\title{
MDM2 SNP309 and risk of cervical cancer
}

\author{
Stian Knappskog • Per Eystein Lønning
}

Received: 26 February 2014 / Accepted: 28 March 2014 / Published online: 17 April 2014

(C) International Society of Oncology and BioMarkers (ISOBM) 2014

\section{Dear Editor,}

We read with interest the paper "MDM2 SNP309 variation increases cervical cancer risk among Asians" by Zhuo et al. in Tumor Biology [1].

This paper assesses the impact of the MDM2 promoter SNP309 (rs2279744) on risk of cervical cancer through a meta-analysis of four original articles (five independent case-control designed studies). The overall data presented by the authors indicate that the SNP309G-allele is not associated with an increased risk of cervical cancer. However, when restricting their analysis to Asian cohorts, the authors find a significantly increased risk of cervical cancer among individuals harbouring the SNP309GG genotype versus individuals with the SNP309TG or TT genotypes. The remaining cohorts (one Caucasian and one cohort of mixed ethnicity) yielded nonsignificant results. Notably, such a difference with respect to ethnicity is similar to what others have observed previously in other cancer forms $[2,3]$. In their discussion, Zhuo et al. state that their observations indicate interactions between the MDM2 polymorphism and ethnic variations. Further, they point to factors such as age, smoking and HPV-status as nonassessed potential confounding factors. While the data presented and the authors' discussion are interesting, we would like to make some additional comments.

S. Knappskog $(\bowtie) \cdot$ P. E. Lønning

Section of Oncology, Department of Clinical Science,

University of Bergen, Bergen, Norway

e-mail: stian.knappskog@med.uib.no

S. Knappskog • P. E. Lønning

Department of Oncology, Haukeland University Hospital, Bergen, Norway
A clear confounding factor relates to a second MDM2 promoter SNP, SNP285G $>$ C (rs117039649), located only 24 bps upstream of SNP309. The C-allele of SNP285 has been shown to have an antagonistic effect versus SNP309G: while in vitro studies have shown the SNP309G-allele to elongate a binding site for the transcription factor $\mathrm{Sp} 1$ and thereby increase MDM2 transcription [4, 5], the SNP285C-allele has the opposite effect, significantly reducing $\mathrm{Sp} 1$ binding [5]. The SNP285C/309G haplotype accounts for about $12 \%$ of all SNP309G alleles in $~ 3,500$ North Western Europeans (Norway, the Netherlands and the UK) [6], and a similar frequency is reported in the Caucasian cohorts of the 1000 genomes project (www.1000genomes.org). Notably, the SNP285C-allele has been found to be absent in Asian (Chinese and Japanese) populations both in our studies [5] and in the Chinese and Japanese cohorts of the 1000 genomes project.

While no study so far has addressed odds ratio for cervical cancer with respect to SNP285 status, notably, this variant has been associated with a significantly reduced risk of cancer of the breast, ovaries and endometrium [5, 7]. Further, the odds ratios for these cancers, related to SNP309G, increased when individuals harbouring the SNP285C variant were censored from the analyses $[5,7]$. If the SNP285C-allele has a protective effect against cervical cancer resembling what has been recorded for the three malignancies mentioned above, this may explain the discrepancy with respect to SNP309 status and risk of cervical cancer between Asian and non-Asian cohorts, as recorded by Zhuo et al.

Taken together, the interesting data presented by Zhuo et al. underlines the importance of determining SNP285 status and correcting for this variant whenever addressing Odds Ratios for cancer related to SNP309 status across different ethnic cohorts. 


\section{References}

1. Zhuo X, Ren J, Li D, Wu Y, Zhou Q. Mdm2 snp309 variation increases cervical cancer risk among Asians. Tumour Biol: J Int Soc Oncodev Biol Med. 2014. doi:10.1007/s13277-014-1695-5.

2. Economopoulos KP, Sergentanis TN. Differential effects of mdm2 snp309 polymorphism on breast cancer risk along with race: a meta-analysis. Breast Cancer Res Treat. 2010;120: 211-6.

3. Hu Z, Jin G, Wang L, Chen F, Wang X, Shen H. Mdm2 promoter polymorphism snp309 contributes to tumor susceptibility: evidence from 21 case-control studies. Cancer Epidemiol Biomarkers Prev. $2007 ; 16: 2717-23$.
4. Bond GL, Hu W, Bond EE, Robins H, Lutzker SG, Arva NC, et al. A single nucleotide polymorphism in the $\mathrm{mdm} 2$ promoter attenuates the p53 tumor suppressor pathway and accelerates tumor formation in humans. Cell. 2004;119:591-602.

5. Knappskog S, Bjornslett M, Myklebust LM, Huijts PE, Vreeswijk MP, Edvardsen $\mathrm{H}$, et al. The mdm2 promoter snp285c/309 g haplotype diminishes sp1 transcription factor binding and reduces risk for breast and ovarian cancer in Caucasians. Cancer Cell. 2011;19:273-82.

6. Knappskog S, Lonning PE. Mdm2 promoter snp285 and snp309; phylogeny and impact on cancer risk. Oncotarget. 2011;2:251-8.

7. Knappskog S, Trovik J, Marcickiewicz J, Tingulstad S, Staff AC, MoMa TECsg, et al. Snp285c modulates oestrogen receptor/sp1 binding to the $\mathrm{mdm} 2$ promoter and reduces the risk of endometrial but not prostatic cancer. Eur J Cancer. 2012;48:1988-96. 